\title{
Generalized Hirsch h-index for disclosing latent facts in citation networks
}

\author{
ANTONIS Sidiropoulos ${ }^{\mathrm{a}}$ Dimitrios KATSAROs, ${ }^{\mathrm{a}, \mathrm{b}}$ YANNIS MANOLOPOULOS ${ }^{\mathrm{a}}$ \\ a Informatics Department, Aristotle University, Thessaloniki (Greece) \\ ${ }^{\mathrm{b}}$ Computer \& Communications Engineering Department, University of Thessaly, Volos (Greece)
}

\begin{abstract}
What is the value of a scientist and its impact upon the scientific thinking? How can we measure the prestige of a journal or a conference? The evaluation of the scientific work of a scientist and the estimation of the quality of a journal or conference has long attracted significant interest, due to the benefits by obtaining an unbiased and fair criterion. Although it appears to be simple, defining a quality metric is not an easy task. To overcome the disadvantages of the present metrics used for ranking scientists and journals, J. E. Hirsch proposed a pioneering metric, the now famous h-index. In this article we demonstrate several inefficiencies of this index and develop a pair of generalizations and effective variants of it to deal with scientist ranking and publication forum ranking. The new citation indices are able to disclose trendsetters in scientific research, as well as researchers that constantly shape their field with their influential work, no matter how old they are. We exhibit the effectiveness and the benefits of the new indices to unfold the full potential of the h-index, with extensive experimental results obtained from the DBLP, a widely known on-line digital library.
\end{abstract}

\section{Introduction}

The evaluation of the scientific work of a scientist has long attracted significant interest, due to the benefits by obtaining an unbiased and fair criterion. Having defined such a metric we can use it for faculty recruitment, promotion, prize awarding, funding allocation, comparison of personal scientific merit, etc. Similarly, the estimation of a

Received September 15, 2006

Address for correspondence:

DIMITRIOS KATSAROS

Aristotle University, Thessaloniki, 54124, Greece

E-mail: dimitris@delab.csd.auth.gr

0138-9130/US \$20.00

Copyright (C) 2007 Akadémiai Kiadó, Budapest

All rights reserved 
publication forum's (journal or conference) quality is of particular interest, since it impacts the scientists' decisions about where to publish their work, the researchers' preference in seeking for important articles, and so on.

Although, the issue of ranking a scientist or a journal/conference dates back to the seventies with the seminal work of Eugene Garfield ${ }^{16}$ and continued with sparse publications, ${ }^{18,20}$ during the last five years we have witnessed a blossom of this field $4,6,7,21,22,25-28,31,35$ due to the proliferation of digital libraries, which made available a huge amount of bibliographic data.

Until present there are two major popular ways for evaluating scientific work and a hybrid of them. The first method is by allowing some contacted experts to perform the ranking and the second method is based on what is termed citation analysis, which involves examining the referring articles of an item (scientist/journal/conference). An amalgamation of them is also possible, although it is more close to the latter approach.

The first method adopts an ad hoc approach, which works by collecting the opinion of different experts (or not) in a domain. The study reported in Ref. 26 focused in the area of Information Systems and performed an on-line survey for 87 journals with 1000 respondents approximately, whereas the authors of Ref. 25 conducted the most extensive survey to date of IS journal rankings. They collected responses from 2559 respondents (32\% of the 8741 targeted faculty members in 414 IS departments worldwide). Instead of using a predetermined journal list, they asked the respondents to freely nominate their top-four research journals. This kind of works is very interesting, because they perform a ranking according to readers' (and authors') perception, which is not always adequately expressed through citation analysis, but they suffer from the fact of being basically "manual" sometimes biased, and not highly computerized (automated) and objective.

On the other hand, the second way of evaluating the scientific work is by defining an objective function that calculates some "score" for the "objects" under evaluation, taking into account the graph structure created by the citations among the published articles. Defining a quality and representative metric is not an easy task, since it should account for the productivity of a scientist and the impact of all of his/her work (analogously for journals/conferences). Most of the existing methods up-to-date are based on some form of (arithmetics upon) the total number of authored papers, the average number of authored papers per year, the total number of citations, the average number of citations per paper, the average number of citations per year, etc. A comprehensive description for many of them can be found in Ref. 36.

Finally, characteristic works implementing the hybrid approach of combining the experts' judge and citation analysis are described in Ref. 22, 38. Their rankings are realized by taking some averages upon the results obtained from the citation analysis and experts' opinion, thus implementing a post-processing step of the two major approaches. 


\section{Motivation for new citation indices}

Although there is no clear winner among citation analysis and experts' assessment, the former method is usually the preferred method, because it can be performed in a fully automated and computerized manner and it is able to exploit the wealth of citation information available in digital libraries.

All the metrics used so far in citation analysis, even those which are based on popular spectral techniques, like HITS, ${ }^{23}$ PageRank $^{29}$ and its variations for bibliometrics, like Ref. 8, present one or more of the following drawbacks (see also Ref. 19:

- They do not measure the importance or impact of papers, e.g., the metrics based solely on the total number of papers.

- They are affected by a small number of "big hits" articles, which received huge number of citations, whereas the rest of the articles may have negligible total impact, e.g., the metrics based on the total number of citations.

- They can not measure productivity, e.g., the metrics based on the average number of citations per paper.

- They have difficulty to set administrative parameters, e.g., the metrics based on the number $x$ of articles, which have received $y$ citations each, or the metrics based on the number $z$ of the most cited articles.

To collectively overcome all these disadvantages of the present metrics, in 2005 J. E. Hirsch proposed the pioneering h-index, ${ }^{19}$ which, in a short period of time, became extremely popular. ${ }^{*}$ The h-index is defined as follows:

Definition 1. A researcher has $h$-index $h$, if $h$ of his/her $N_{p}$ articles have received at least $h$ citations each, and the rest $\left(N_{p}-h\right)$ articles have received no more than $h$ citations. ${ }^{1,19}$

This metric calculates how broad the research work of a scientist is. The h-index accounts for both productivity and impact. For some researcher, to have large h-index, s/he must have a lot of "good" articles, and not just a few "good" articles.

The h-index acts as a lower bound on the real number of citations for a scientist. In fact, there is a significant gap between the total number of citations as calculated by $\mathrm{h}$ index and the real number of citations of a scientist. Think that the quantity $h$ will always be smaller than or equal to the number $N_{p}$ of the articles of a researcher; it holds that $h^{2} \leq N_{c, t o t}$, where $N_{c, t o t}$ is the total number of citations that the researcher has

\footnotetext{
* Notice that the economics literature defines the $H$ index (the Herfindahl-Hirschman index), which is a way of measuring the concentration of market share held by particular suppliers in a market. The $H$ index is the sum of squares of the percentages of the market shares held by the firms in a market. If there is a monopoly, i.e., one firm with all sales, the $H$ index is 10000 . If there is perfect competition, with an infinite number of firms with near-zero market share each, the $H$ index is approximately zero. Other industry structures will have $H$ indices between zero and 10000 .
} 
received. Apparently, the equality holds when all the articles, which contribute to $\mathrm{h}$ index have received exactly $h$ citations each, which is quite improbable. Therefore, in the usual case it will hold that $h^{2}<N_{c, t o t}$. To bridge this gap, J. E. Hirsch defined the index $a$ as follows:

Definition 2. A scientist has a-index a if the following equation holds: 19

$$
N_{c, t o t}=a h^{2}
$$

The a-index can be used as a second metric-index for the evaluation and ranking of scientists. It describes the "magnitude" of each scientist's "hits". A large $a$ implies that some article(s) have received a fairly large number of citations compared to the rest of its articles and with respect to what the $\mathrm{h}$-index presents.

The introduction of the $\mathrm{h}$-index was a major breakthrough in citation analysis. Though several aspects of the inefficiency of the original h-index are apparent; or to state it in its real dimension, significant efforts are needed to unfold the full potential of h-index. Firstly, the original h-index assigns the same importance to all citations, no matter what their age is, thus refraining from revealing the trendsetters scientists. Secondly, the h-index assigns the same importance to all articles, thus making the young researchers to have a relatively small h-index, because they did not have enough time either to publish a lot of good articles, or time to accumulate large number of citation for their good papers. Thus, the h-index can not reveal the brilliant though young scientists.

\section{Our contributions}

The purpose of our work is to extend and generalize the original h-index in such ways, so as to reveal various latent though strong facts hidden in citation networks. Our proposals aim to maintain the elegance and ease of computation of the original h-index, thus we strive for developing relatively simple indexes, since we believe that the simplicity of the h-index is one of its beauties. In this context, the article makes the following contributions:

- Introduces two generalizations of the h-index, namely the contemporary $h$-index and the trend h-index, which are appropriate for scientist ranking and are able to reveal brilliant young scientists and trendsetters, respectively. These two generalizations can also be used for the cases of conference and journal ranking.

- Introduces a normalized version of the h-index for scientist ranking, namely the normalized h-index.

- Introduces two variants of the $\mathrm{h}$-index appropriate for journal/conference ranking, namely the yearly $h$-index and the normalized yearly $h$-index. 
- Performs an extensive experimental evaluation of the aforementioned citation indices, using real data from DBLP, an online bibliographic database.

Developing mathematical models and conducting theoretical analysis of the properties of the proposed indices is the next step in this work, but it is beyond the scope of this paper; here we are interested in providing extensive experimental evidence of the power of the generalizations to the h-index.

\section{Novel citation indices for scientist ranking}

After the introduction of the h-index, a number of other proposals followed, either presenting case studies using it, $3,5,10,11,30,32,34$ or describing a new variation of it ${ }^{13,33}$ (aiming to bridge the gap between the lower bound of the total number of citations calculated by h-index and their real number), or presenting normalized versions of it with respect to the time-span, 2,44 or studying its mathematics and its performance. $9,12,14,17$

Deviating from their line of research, we develop in this article a pair of generalizations of the $\mathrm{h}$-index for ranking scientists, which are novel citation indices, a normalized variant of the $\mathrm{h}$-index and a pair of variants of the $\mathrm{h}$-index suitable for journal/conference ranking.

\section{The contemporary h-index}

The original h-index does not take into account the "age" of an article. It may be the case that some scientist contributed a number of significant articles that produced a large h-index, but now s/he is rather inactive or retired. Therefore, senior scientists, who keep contributing nowadays, or brilliant young scientists, who are expected to contribute a large number of significant works in the near future but now they have only a small number of important articles due to the time constraint, are not distinguished by the original h-index. Thus, it arises the need of defining a generalization of the h-index, in order to account for these facts.

We define a novel score $S^{c}(i)$ for an article $i$ based on citation counting, as follows:

$$
S^{c}(i)=\gamma *(Y(\text { now })-Y(i)+1)^{-\delta} *|C(i)|
$$

where $Y(i)$ is the publication year of an article $i$ and $C(i)$ are the articles citing the article $i$. If we set $\delta=1$, then $S^{c}(i)$ is the number of citations that the article $i$ has received, divided by the "age" of the article. Since we divide the number of citations with the time interval, the quantities $S^{c}(i)$ will be too small to create a meaningful h-index; thus, we use the coefficient $\gamma$. In our experiments reported in the Experiments sections, we use the value of 4 for the coefficient $\gamma$. Thus, for an article published during the current 
year, its citations account four times. For an article published 4 year ago, its citations account only one time. For an article published 6 year ago, its citations account $4 / 6$ times, and so on.

This way, an old article gradually loses its "value", even if it still gets citations. In other words, in the calculations we mainly take into account the newer articles." Therefore, we define a novel citation index for scientist rankings, the contemporary $\mathrm{h}$ index, expressed as follows:

Definition 3. A researcher has contemporary $h$-index $h^{c}$, if $h^{c}$ of its $N_{p}$ articles get a score of $S^{c}(i) \geq h^{c}$ each, and the rest $\left(N_{p}-h^{c}\right)$ articles get a score of $S^{c}(i) \leq h^{c}$.

\section{The trend h-index}

The original h-index does not take into account the year when an article acquired a particular citation, i.e., the "age" of each citation. For instance, consider a researcher who contributed to the research community a number of really brilliant articles during the decade of 1960, which, say, got a lot of citations. This researcher will have a large $\mathrm{h}$-index due to the works done in the past. If these articles are not cited anymore, it is an indication of an outdated topic or an outdated solution. On the other hand, if these articles continue to be cited, then we have the case of an influential mind, whose contributions continue to shape newer scientists' minds. There is also a second very important aspect in aging the citations. There is the potential of disclosing trendsetters, i.e., scientists whose work is considered pioneering and sets out a new line of research that currently is hot ("trendy"), thus this scientist's works are cited very frequently.

To handle this case, we take the opposite approach than the contemporary h-index's; instead of assigning to each scientist's article a decaying weight depending on its age, we assign to each citation of an article an exponentially decaying weight, which is expressed as a function of the "age" of the citation. This way, we aim at estimating the impact of a researcher's work in a particular time instance. We are not interested in how old the articles of a researcher are, but whether they still get citations. We define an equation similar to Equation 2, which is expressed as follows:

$$
S^{t}(i)=\gamma * \sum_{\forall x \in C(i)}(Y(\text { now })-Y(x)+1)^{-\delta}
$$

where $\gamma, \delta, Y(i)$ and $S(i)$ for an article $i$ are as defined earlier. We define a novel citation index for scientist ranking, the trend $\mathrm{h}$-index, expressed as follows:

Definition 4. A researcher has trend $h$-index $h^{t}$, if $h^{t}$ of its $N_{p}$ articles get a score of $S^{t}(i) \geq h^{t}$ each, and the rest $\left(N_{p}-h^{t}\right)$ articles get a score of $S^{t}(i) \leq h^{t}$ each.

\footnotetext{
${ }^{*}$ Apparently, if $\delta$ is close to zero, then the impact of the time penalty is reduced, and, for $\delta=0$, this variant coincides with the original h-index for $\gamma=1$.
} 
Apparently, for $\gamma=1, \delta=0$, the trend h-index coincides with the original h-index.

It is straightforward to devise a generalization of both the contemporary h-index and the trend h-index, which takes into account both the age of a scientist's article and the age of each citation to this article, but such index does not provide many additional insights about the real contributions of a scientist. Therefore, we do not investigate further this generalization in the present article.

\section{The normalized $h$-index}

Since the scientists do not publish the same number of articles, the original $h$-index is not the fairer metric; thus, we define a normalized version of $h$-index, expressed as follows:

Definition 5. A researcher has normalized $h$-index $h^{n}=h / N_{p}$, if $h$ of its $N_{p}$ articles have received at least $h$ citations each, and the rest $\left(N_{p}-h\right)$ articles received no more than $h$ citations.

In the next section, we define some variants of the h-index family of citation indices for ranking journals/conferences.

\section{New citation indices for journals and conferences ranking}

Based on the original idea of the $\mathrm{h}$-index and on the aforementioned generalizations and variants, we define analogous concepts for ranking journals and conferences. For instance, the h-index of a journal/magazine or a conference is $h$, if $h$ of the $N_{p}$ articles that contains, have received at least $h$ citations each, and the rest $\left(N_{p}-h\right)$ articles received no more than $h$. The generalizations of contemporary h-index and trend h-index can be defined for conferences and journals as well, similarly to the Definitions 3 and 4. Direct applications of the h-index in journal ranking following this definition appeared in Refs 3, 10, 32. Though we observe that the direct application of the index can not guarantee a fair comparison between conferences or between journals, because a) their lives are different, and $b$ ) they publish different numbers of articles.

We deal with the first problem by calculating the h-index on a per year basis. In particular, we define that:

Definition 6. A conference or a journal has yearly h-index $h_{y}$ for the year $y$ if $h_{y}$ of its articles $N_{p, y}$ published during the year $y$ have received at least $h_{y}$ citations each, and the rest $\left(N_{p, y}-h_{y}\right)$ articles received no more than $h_{y}$ citations.

For instance, the $\mathrm{h}$-index for the year 1992 , denoted as $h_{1992}$, of the VLDB conference is computed as the number of its articles which have received more than $h_{1992}$ citations. The drawbacks though of the aforementioned metric are the following:

1. The conferences/journals do not publish exactly the same number of articles. Thus, for a conference which published around 50 articles, the 
upper bound for its h-index is 50 . Another conference which published 150 , the upper bound for its h-index is 150 and it also has much more stronger probability to exceed the limit of 50 . The number of articles appearing in a year in a conference or journal reflects the preference of the researchers to this publication forum. If we consider that the forum published 50 articles, because it could not attract more valuable articles, then it correctly has as upper bound the number 50 and it is not a problem that it can not overrule forum $B$. On the other hand, perhaps we are interested in the average "quality" of the articles published in a forum, no matter what the number of published articles in a forum is.

2. The $h_{y}$ index constantly changes. Even though we examine a conference which took place in 1970, the $h_{y}$ index that we can calculate today, is possible to change a few years later. Thus, the drawback of this index is that we can not have a final evaluation for the forums of a year, no matter how old they are.

The only way to overcome the second drawback is to add a time window after the organization of a conference or the publication of a journal (i.e., ten or five years time window). This would add the notion of the Impact Factor ${ }^{37}$ to the metric, which is beyond the scope of our current research.

To address the first drawback, we define a "parallel" index, which is normalized with respect to the number of articles published in a forum. Its formal definition is given below:

Definition 7. A conference or journal for the year $y$ has normalized index $h_{y}^{n}=h_{y} / N_{p, y}$, if $h_{y}$ of its $N_{p, y}$ articles in the year $y$ have received at least $h_{y}$ citations each, and the rest $\left(N_{p, y}-h_{y}\right)$ articles received no more than $h_{y}$ citations.

Having defined these generalizations and variants of the original h-index, we will evaluate in the subsequent sections their success in identifying scientists or forums with extraordinary performance or their ability to reveal latent facts in a citation network, such as brilliant young scientists and trendsetters. For the evaluation, we will exploit the on-line database of DBLP.*

\section{Experiments}

In this section we will present the ranking results for scientists, conferences and journals by using the basic h-index definition as well as by using the generalizations and variants developed in the previous sections. Along the lines of Refs 36-38, our dataset consists of the DBLP collection (DBLP timestamp: Mar/3/2006). The reasons

\footnotetext{
* The DBLP digital library with bibliographic data on "Databases and Logic Programming" is maintained by Michael Ley at the University of Trier, accessible from http://dblp.uni-trier.de/
} 
for selecting this source of data instead of ISI or Google Scholar or CiteSeer are the following:

1. DBLP contains data about journal and conference publications as well.

2. DBLP data are focused mostly in the area of Databases.

3. The maintainers of DBLP library put a lot of work into resolving the "names problem" - the same person referenced with (many) different names.

4. DBLP's data format gave us the possibility of eliminating "self-citations", which is not done by Google Scholar.

5. Google Scholar only takes into account the papers that finds on the Web.

6. ISI's coverage for computer science is not comprehensive.

7. CiteSeer does not eliminate "self-citations", does not rank author queries by citation number and also weights them by year.

It is worthwhile noticing that many top conferences of this area are very competitive (with an acceptance ratio stronger than 1:3 and up to 1:7), and occasionally more competitive than the top journals of the area. In many computer science departments worldwide, publications in these conferences are favored in comparison to journal publications. Therefore, a ranking of conferences on databases is equally important to the ranking of the journals of the area.

The used database snapshot contains 451694 inproceedings, 266307 articles, 456511 authors, 2024 conference series and 504 journals. Also, the number of citations in our dataset is 100205 . Although this number is relatively small, it is a satisfactory sample for our purposes. Almost all citations in the database are made from publications prior to the year 2001. Thus, we can assume that the results presented here correspond to the year 2001. From now on, with the term "now" we actually mean sometime near 2001. Although other bibliographic sources (e.g., ISI, Google Scholar, CiteSeer) are widely available, the used collection has the aforementioned desired characteristics and thus it is sufficient for exhibiting the benefits of our proposed citation indices, without biasing our results.

\section{Experiments with the h-index for scientists}

In Tables 1, 2, 3 and 4 we present the resulting ranking using the h-index, as well as its defined generalizations. In these tables columns $a_{c}$ and $a_{t}$ stand for the a-index corresponding to the contemporary h-index and trend h-index, respectively. The computation of $a_{c}$ (and $a_{t}$ ) is analogous to the original a-index computation, but it uses the functions $S_{c}(i)$ and $S_{c}(i)$ defined earlier for all the publications of each author, rather 
than using the total number of citations. ${ }^{*}$ This is depicted in Equations 4 and 5, where $P$ is the set of author's publications.

$$
\begin{gathered}
\sum_{\forall i \in P} S_{c}(i)=a_{c} h_{c}^{2} \\
\sum_{\forall i \in P} S_{t}(i)=a_{t} h_{t}^{2}
\end{gathered}
$$

Based on the contemporary h-index and trend h-index definitions, it is obvious that the conditions $\sum_{\forall i \in P} S_{c}(i) \geq h_{c}^{2}$ and $\sum_{\forall i \in P} S_{t}(i) \geq h_{t}^{2}$ are true. The equality holds only in the unusual case that every $S_{c}(i)$ is equal to $h_{c}$.

At a first glance, we see that the values computed for h-index (Table 1) are much lower than the values presented in Ref. 19 for physics scientists due to the non completeness of the source data. We can also notice that the values for $h, h_{c}$ and $h_{t}$ are different from each other as well as there are differences in the ordering of the scientists. This confirms our allegation for the convenience of these indices and will be discussed in the sequel of the article.

\begin{tabular}{lccrr}
\multicolumn{5}{c}{ Table 1. Scientist ranking using the h-index } \\
\hline \multicolumn{1}{c}{ Name } & $h$ & \multicolumn{1}{c}{$a$} & $N_{c, \text { tot }}$ & $N_{p}$ \\
\hline 1. Michael Stonebraker & 24 & 3.78 & 2180 & 193 \\
2. Jeffrey D. Ullman & 23 & 3.37 & 1783 & 227 \\
3. David J. DeWitt & 22 & 3.91 & 1896 & 150 \\
4. Philip A. Bernstein & 20 & 3.39 & 1359 & 124 \\
5. Won Kim & 19 & 2.96 & 1071 & 143 \\
6. Catriel Beeri & 18 & 3.16 & 1024 & 93 \\
7. Rakesh Agrawal & 18 & 3.06 & 994 & 154 \\
8. Umeshwar Dayal & 18 & 2.81 & 913 & 130 \\
9. Hector Garcia-Molina & 17 & 3.60 & 1041 & 314 \\
10. Yehoshua Sagiv & 17 & 3.52 & 1020 & 121 \\
11. Ronald Fagin & 17 & 2.83 & 818 & 121 \\
12. Jim Gray & 16 & 6.13 & 1571 & 118 \\
13. Serge Abiteboul & 16 & 4.33 & 1111 & 172 \\
14. Michael J. Carey & 16 & 4.25 & 1090 & 151 \\
15. Nathan Goodman & 16 & 3.37 & 865 & 68 \\
16. Christos Faloutsos & 16 & 2.89 & 742 & 175 \\
17. Raymond A. Lorie & 15 & 6.23 & 1403 & 35 \\
18. Jeffrey F. Naughton & 15 & 2.90 & 653 & 123 \\
19. Bruce G. Lindsay & 15 & 2.76 & 623 & 60 \\
20. David Maier & 14 & 5.56 & 1090 & 158 \\
\hline
\end{tabular}

\footnotetext{
${ }^{*}$ Notice here that the equations $N_{c, t o t}=a_{c} h_{c}^{2}$ and $N_{c, t o t}=a_{t} h_{t}^{2}$ are not true since $\sum_{\forall i \in P} S_{c}(i)$ and $\sum_{\forall i \in P} S_{t}(i)$ are different than $N_{c, t o t}$.
} 
A superficial examination of Tables 2 and 3 does not reveal any major difference between their ranking and the ranking obtained by h-index (in Table 1). With respect to Table 2, the astute reader though, will catch three important representative cases: the case of Christos Faloutsos, the case of Serge Abiteboul and the case of Jennifer Widom. Christos Faloutsos is at the $16^{\text {th }}$ place of h-index table. In contemporary h-index table he climbs to the $14^{\text {th }}$ position. Serge Abiteboul climbs up from the $13^{\text {th }}$ position to the $5^{\text {th }}$ position. Similarly, Jennifer Widom appears in the $6^{\text {th }}$ position of the contemporary h-index (Table 2), although she does not have an entry in the h-index (see Table 1). This means that the major amount of their good publications is published in the recent years (relatively to the rest of the scientists). In other words, they work on now hot topics. Consequently, we would characterize their works as contemporary.

Table 2. Scientist ranking using the contemporary h-index

\begin{tabular}{lccccc}
\hline \multicolumn{1}{c}{ Name } & $h_{c}$ & $a_{c}$ & $h$ & $N_{c, t o t}$ & $N_{p}$ \\
\hline 1.David J. DeWitt & 14 & 3.10 & 22 & 1896 & 150 \\
2. Jeffrey D. Ullman & 13 & 3.44 & 23 & 1783 & 227 \\
3. Michael Stonebraker & 12 & 3.98 & 24 & 2180 & 193 \\
4. Rakesh Agrawal & 12 & 3.24 & 18 & 994 & 154 \\
5. Serge Abiteboul & 11 & 4.08 & 16 & 1111 & 172 \\
6.Jennifer Widom & 11 & 3.23 & 14 & 709 & 136 \\
7.Jim Gray & 10 & 3.93 & 16 & 1571 & 118 \\
8. Michael J. Carey & 10 & 3.79 & 16 & 1090 & 151 \\
9. Won Kim & 10 & 3.00 & 19 & 1071 & 143 \\
10.David Maier & 10 & 2.93 & 14 & 1090 & 158 \\
11.Hector Garcia-Molina & 9 & 5.30 & 17 & 1041 & 314 \\
12.Jeffrey F. Naughton & 9 & 3.85 & 15 & 653 & 123 \\
13. Yehoshua Sagiv & 9 & 3.76 & 17 & 1020 & 121 \\
14. Christos Faloutsos & 9 & 3.68 & 16 & 742 & 175 \\
15. Catriel Beeri & 9 & 3.59 & 18 & 1024 & 93 \\
16.Philip A. Bernstein & 9 & 3.49 & 20 & 1359 & 124 \\
17.Umeshwar Dayal & 9 & 3.39 & 18 & 913 & 130 \\
18. Hamid Pirahesh & 9 & 3.34 & 14 & 622 & 67 \\
19.H. V. Jagadish & 9 & 2.88 & 12 & 503 & 151 \\
20. Raghu Ramakrishnan & 8 & 5.05 & 14 & 818 & 147 \\
\hline
\end{tabular}

The results appear more impressive in the trend h-index (Table 3). Christos Faloutsos climbs to the $8^{\text {th }}$ position and Jennifer Widom in the $5^{\text {th }}$ position. This shows that their publications get citations during the very recent years. Consequently, we would characterize the work of Faloutsos and Widom as "trendy", in the sense that a general interest exists by the rest of the research community for the work of the specific scientists during the particular time period. Indeed, Faloutsos is recognized as (one of) the main trendsetter in the area of spatial, multidimensional and time series data management. Widom is recognized as (one of) the main trendsetter in the area of semistructured data management. Figures 1a and 1e, which will be examined in the sequel, reveal that the main volume of the total citations to these scientists started at specific years, when they contributed significant ground-setting papers in their domains. 
A. SiDIROPOULOS et al.: Generalized Hirsch h-index

Table 3. Scientist ranking using the trend h-index

\begin{tabular}{lccccc}
\hline \multicolumn{1}{c}{ Name } & $h_{t}$ & $a_{t}$ & $h$ & $N_{c, t o t}$ & $N_{p}$ \\
\hline 1.David J. DeWitt & 20 & 2.73 & 22 & 1896 & 150 \\
2. Michael Stonebraker & 17 & 3.61 & 24 & 2180 & 193 \\
3.Jeffrey D. Ullman & 17 & 3.45 & 23 & 1783 & 227 \\
4. Rakesh Agrawal & 17 & 3.06 & 18 & 994 & 154 \\
5.Jennifer Widom & 16 & 2.81 & 14 & 709 & 136 \\
6. Serge Abiteboul & 14 & 4.07 & 16 & 1111 & 172 \\
7.Hector Garcia-Molina & 14 & 4.03 & 17 & 1041 & 314 \\
8. Christos Faloutsos & 14 & 3.15 & 16 & 742 & 175 \\
9.Jim Gray & 13 & 4.46 & 16 & 1571 & 118 \\
10.Jeffrey F. Naughton & 13 & 3.36 & 15 & 653 & 123 \\
11. Won Kim & 13 & 3.23 & 19 & 1071 & 143 \\
12. Michael J. Carey & 12 & 4.79 & 16 & 1090 & 151 \\
13. Yehoshua Sagiv & 12 & 3.96 & 17 & 1020 & 121 \\
14.Umeshwar Dayal & 12 & 3.41 & 18 & 913 & 130 \\
15. Catriel Beeri & 12 & 3.12 & 18 & 1024 & 93 \\
16. Raghu Ramakrishnan & 11 & 4.41 & 14 & 818 & 147 \\
17.Philip A. Bernstein & 11 & 4.03 & 20 & 1359 & 124 \\
18.David Maier & 11 & 3.94 & 14 & 1090 & 158 \\
19.Hamid Pirahesh & 11 & 3.87 & 14 & 622 & 67 \\
20.H. V. Jagadish & 11 & 3.58 & 12 & 503 & 151 \\
\hline
\end{tabular}

Table 4. Scientist ranking using the normalized h-index

\begin{tabular}{lccccc}
\hline \multicolumn{1}{c}{ Name } & $h_{n}$ & $h$ & $a$ & $N_{c, \text { tot }}$ & $N_{p}$ \\
\hline 1. Rajiv Jauhari & 1 & 5 & 3.72 & 93 & 5 \\
2.Jie-Bing Yu & 1 & 5 & 2.36 & 59 & 5 \\
3.L. Edwin McKenzie & 1 & 5 & 2.04 & 51 & 5 \\
4. Upen S. Chakravarthy & 0.88 & 8 & 2.60 & 167 & 9 \\
5. James B. Rothnie Jr. & 0.85 & 6 & 6.55 & 236 & 7 \\
6. M. Muralikrishna & 0.85 & 6 & 5.47 & 197 & 7 \\
7. Stephen Fox & 0.83 & 5 & 4.12 & 103 & 6 \\
8. Antonin Guttman & 0.8 & 4 & 20.43 & 327 & 5 \\
9. Marc G. Smith & 0.8 & 4 & 4.81 & 77 & 5 \\
10. Gail M. Shaw & 0.8 & 4 & 4.37 & 70 & 5 \\
11. Glenn R. Thompson & 0.8 & 4 & 4.37 & 70 & 5 \\
12.David W. Shipman & 0.75 & 6 & 11.16 & 402 & 8 \\
13.Dennis R. McCarthy & 0.75 & 6 & 5.30 & 191 & 8 \\
14. Spyros Potamianos & 0.66 & 4 & 10.43 & 167 & 6 \\
15. Robert K. Abbott & 0.66 & 4 & 4.68 & 75 & 6 \\
16. Edward B. Altman & 0.66 & 4 & 3.06 & 49 & 6 \\
17. Brian M. Oki & 0.66 & 4 & 2.56 & 41 & 6 \\
18. Gene T. J. Wuu & 0.66 & 6 & 2.25 & 81 & 9 \\
19. Marguerite C. Murphy & 0.66 & 4 & 1.62 & 26 & 6 \\
20. Gerald Held & 0.62 & 5 & 9.84 & 246 & 8 \\
\hline
\end{tabular}

It is also worthwhile to mention that the contemporary h-index and trend h-index are fair metrics for the "all-time classic" scientists, e.g., Jeffrey Ullman, Michael Stonebraker and David DeWitt, whose influential works continue to shape the modern scientists way of thinking.

By examining Tables 2, 3 and 4, we observe that the rankings provided by normalized $\mathrm{h}$-index differ significantly from the rankings provided by the other metrics. 


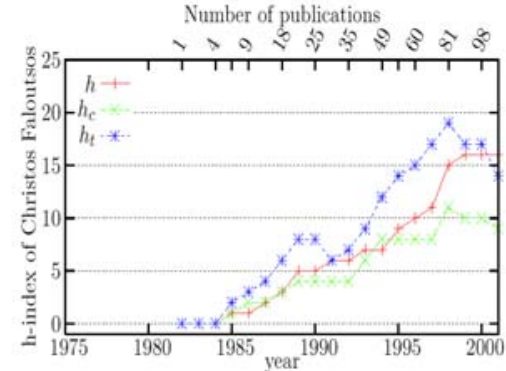

(a)

Number of publications

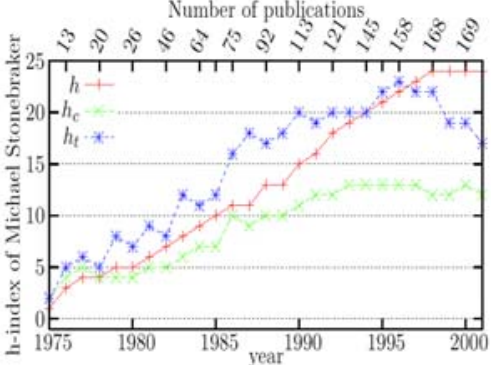

(c)

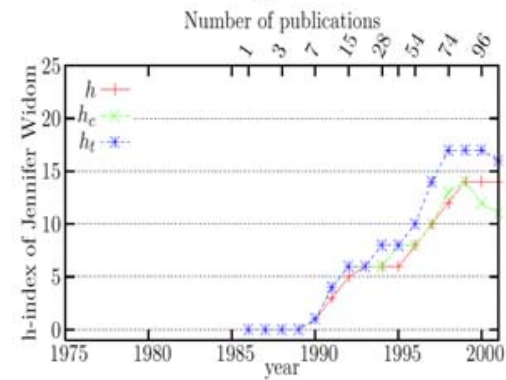

(e)

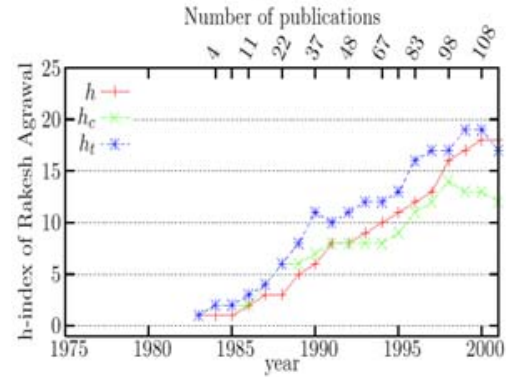

(g)

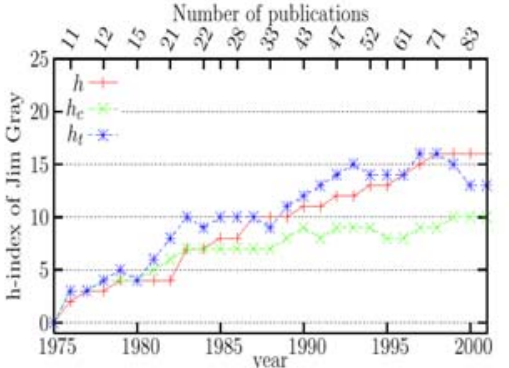

(b)

Number of publications

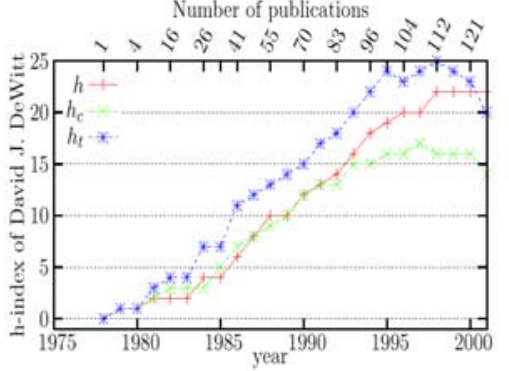

(d)

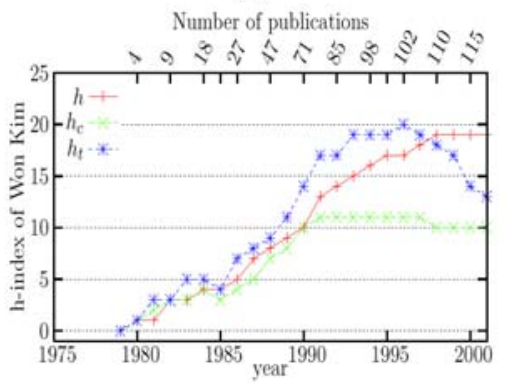

(f)

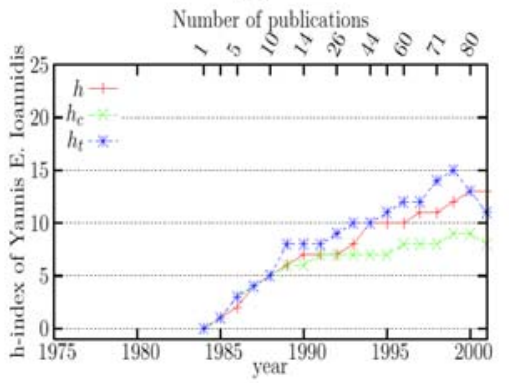

(h)

Figure 1. The h-index of scientists working in databases area 
This happens because the scientists with few but good publications take advantage. Thus, we cannot evaluate the research work of a scientist by taking into consideration only the normalized h-index. The normalized $\mathrm{h}$-index can be used in parallel to $\mathrm{h}$-index and as a second criterion. We can easily assume that the majority of the scientists presented in Table 4 are probably $\mathrm{PhD}$ or MSc students that wrote a fine article with a "famous" professor and after that, they stopped their research career. The second possibility is that the main part of their articles are not included in the DBLP collection - probably because they actually belong to a scientific discipline other than Databases and Logic Programming. Finally, it is always possible to track "promising" researchers among them, who will continue their significant research work.

Motivated by the differences in the above tables, we present the collection of graphs in Figure 1. In these figures we can see the history of the h-index for those scientists, who present significant differences between the h-index family of citation indices, and also those who have a rapid upwards slope at their plot curves. ${ }^{*}$ For each scientist, we provide a justification for the resulting curves related to his/her main research interests. It is the case that these scientists have really broad interests that can not be confined in a single term. Thus, we attempt to characterize the field where they made their most significant contributions.

Comparing Figures 1a and $1 \mathrm{~b}$ regarding Christos Faloutsos and Jim Gray respectively, we can see that the two scientists have the same h-index now. However, Christos Faloutsos has a more ascending slope than Jim Gray, since he started being cited in 1984, while Jim Gray in 1976. Also, the trend h-index $\left(h_{t}\right)$ curve of Christos Faloutsos stays constantly over the h-index $(h)$ equivalent. This means that Christos Faloutsos is getting cited very often at the present and thus, we expect his h-index to get higher than Jim Gray's h-index. Finally, Jim Gray's contemporary h-index $\left(h_{c}\right)$ is constantly below $h$ since 1985 and it diverges with time. This indicates that since 1985 he has not presented exceptionally seminal papers (relatively to his older ones) and after this point the progress is digressive. Indeed, the Turing Award recipient Jim Gray made ground setting contributions to transaction processing; this topic is considered as a solved problem now and it is not considered as an active research area. On the other hand, the main contributions of Christos Faloutsos focus in the area of spatial and multidimensional data management, which became very popular roughly since 1995 .

Figures $1 \mathrm{c}$ and $1 \mathrm{~d}$ correspond to Michael Stonebraker and David J. DeWitt. Both of these researchers are on the top of our list. We can notice that David J. DeWitt' contemporary h-index is very close to his h-index, which means that he keeps publishing very good papers. On the contrary, Michael Stonebraker has started to deflect since 1985. This helps us understand that Michael Stonebraker' trend h-index will also decrease after some years, as it is shown in the same figure. Thus, while

\footnotetext{
* Again, we remind that our data set is rather incomplete for the years after 2000, and thus a downwards pitch for all the researchers appears during the years 1999-2000. However, the results are indicative.
} 
Michael Stonebraker is in higher position than David J. DeWitt at the h-index ranking, David J. DeWitt comes first when examining the other two variations. This means that, if the productivity level of the two researchers keeps on the same pace, the second will soon surpass the first one at the h-index as well.

In Figure 1e we see the progress rate for Jennifer Widom. While Jennifer Widom is not even among the top 20 researchers using the $\mathrm{h}$-index, she is on the $6^{\text {th }}$ and $5^{\text {th }}$ position using the contemporary h-index and trend h-index, respectively. She is the only researcher from our list that presents such a big difference on the timing rates compared to the basic h-index. As we can also see from the diagram, this difference is justifiable, since the increase rate of the basic h-index is high. She is also the only researcher that her contemporary h-index is constantly close to h-index and not below. Finally, although for all the researchers that we present the trend $\mathrm{h}$-index is always lower than the h-index in the year of 2000, the trend h-index remains higher in her case. Jennifer Widom made some ground breaking contributions on building semistructured data management systems, that laid the foundations for the modern XML management systems.

In Figure 1f Won Kim presents an analogous path with Stonebraker. For instance, there is a high ascending curve for trend h-index, but contemporary h-index remains low after 1990 and finally it is obvious that the trend h-index will also follow a decreasing path. Therefore, we expect that h-index will not present high increase. This is explained by the fact that the main research interests of Won Kim was on objectoriented database systems, which flourished during the last years of the eighties and in the first years of the nineties, but it later became a relatively inactive area.

Our observations about Figure 1g concerning the work of Rakesh Aggrawal are analogous to those of Figure 1a concerning Christos Faloutsos, thus we do not go into further details. In Figure $1 \mathrm{~h}$ concerning Yannis Ioannidis, we see an increasing trend that is analogous to that of Jim Gray. The trend h-index remains constantly over hindex, which means that there is a remarkable potential. In addition, the contemporary h-index presents a small deflection from h-index after 1993, which is completely analogous to that of Jim Gray after 1985. Based on the available data, Yannis Ioannidis follows the same progress path as Jim Gray, with a time lag of about 10 years.

Matching the h-index rankings to the awarded scientists. Our next investigation concerns an experiment that tests whether the rankings by the h-index and its generalizations are in accordance with the awards for the database scientific domain. In Refs 36 and 38 we used the 'SIGMOD E. F. Codd Innovations Award' to evaluate some ranking methods. Here, we perform an analogous experiment. The higher an awarded scientist is ranked, it is considered to be the better ranking method. We have to note here, that the issue of awarding a scientist a particular award is not simply a matter of arithmetics (some numerical metrics), but it is very complex. Thus, we use this methodology to test whether the citation indices defined in this metric reveal some general trends. 
Table 5. Citations indices of scientists awarded with SIGMOD E. F. Codd Innovations Award

\begin{tabular}{l|rrr|rrr|r|rrr|}
\hline Name & $h$ & $h_{c}$ & $h_{t}$ & $h$ & $h_{c}$ & $h_{t}$ & year & $h$ & $h_{c}$ & $h_{t}$ \\
\hline Michael Stonebraker & 1 & 3 & 2 & 3 & 2 & 1 & 1992 & 1 & 2 & 1 \\
Jim Gray & 12 & 7 & 9 & 12 & 11 & 10 & 1993 & 15 & 11 & 8 \\
Philip A. Bernstein & 4 & 16 & 17 & 2 & 6 & 4 & 1994 & 2 & 9 & 5 \\
David J. DeWitt & 3 & 1 & 1 & 3 & 1 & 1 & 1995 & 2 & 1 & 1 \\
C. Mohan & 28 & 37 & 31 & 44 & 36 & 35 & 1996 & 49 & 23 & 19 \\
David Maier & 20 & 10 & 18 & 11 & 9 & 15 & 1997 & 15 & 10 & 17 \\
Serge Abiteboul & 13 & 5 & 6 & 17 & 4 & 11 & 1998 & 16 & 6 & 11 \\
Hector Garcia-Molina & 9 & 11 & 7 & 10 & 8 & 4 & 1999 & 14 & 7 & 5 \\
Rakesh Agrawal & 7 & 4 & 4 & 9 & 4 & 4 & 2000 & 7 & 4 & 4 \\
Rudolf Bayer & 145 & 196 & 183 & 142 & 218 & 222 & 2001 & 145 & 196 & 183 \\
Patricia G. Selinger & 143 & 144 & 119 & 143 & 144 & 119 & 2002 & 143 & 144 & 119 \\
Donald D. Chamberlin & 44 & 87 & 69 & 44 & 87 & 69 & 2003 & 44 & 87 & 69 \\
Ronald Fagin & 11 & 39 & 32 & 11 & 39 & 32 & 2004 & 11 & 39 & 32 \\
Lowest Ranking Point & 145 & 196 & 183 & 143 & 218 & 222 & & 145 & 196 & 183 \\
Sum of Rank points & 440 & 560 & 498 & 451 & 543 & 539 & & 464 & 494 & 469 \\
\hline
\end{tabular}

Table 5 presents the list of the awarded scientists by the 'SIGMOD E.F.Codd Innovations Award'. In the first group of columns entitled as $\left(h, h_{c}, h_{t}\right)$ the current position of each scientist is presented by using the respective index. The second triplet of $\left(h, h_{c}, h_{t}\right)$ shows the scientist positions one year before the awarding. Column "year" shows the year of the awarding, whereas the last triplet of $\left(h, h_{c}, h_{t}\right)$ presents his position during (at the end of) the year of the awarding.

Although our data are not complete for the time period after 2000, however, we can make interesting observations for the years before 2000:

- C. Mohan. Although he is ranked relatively low at this moment by using the trend h-index and the contemporary h-index, during the year of 1996 he was ranked higher according to the trend h-index. This was later depicted on the h-index since from the $49^{\text {th }}$ position where he was ranked during 1996, he now climbed up to the $28^{\text {th }}$ position.

- Other similar qualitative cases with obvious difference in the actual ranking are of Hector Garcia-Molina and Philip A. Bernstein.

- Serge Abiteboul. During the year of the awarding the trend h-index is relatively low (compared to the contemporary h-index). According to the contemporary h-index, he was ranked in a higher place. This shows that he had presented interesting work during the age of the awarding; he received the award before his work got reflected to the trend h-index and h-index. Thus, in some cases, the contemporary h-index gives information that it cannot be depicted into the other indices.

- For the cases of Michael Stonebraker and David J. DeWitt, we see that they are stable at the top positions. 


\section{Experiments with conferences and journals ranking}

Experiments with conferences ranking. To evaluate our citation indices on conference ranking, we extract only the database conferences (as defined in Ref. 15) from the data we used in the previous section. In the first part of this section we will make experiments using only the indicators that we fixed for scientists, namely h-index, normalized h-index, contemporary h-index and trend h-index. In Table 6 we present the top-10 conferences using the h-index for the ordering. ${ }^{*}$ Since the quality of the conferences is relatively constant, we observe that in Tables 7 and 8 there are no significant differences in the ranking. The ordering changes dramatically in Table 9 due to the fact that complete data exist only for some conferences.

Table 6. Conferences ranking using the h-index

\begin{tabular}{lcccc}
\hline Name & $h$ & $a$ & $N_{c, \text { tot }}$ & $N_{p}$ \\
\hline 1. sigmod & 45 & 6.05 & 12261 & 2059 \\
2.vldb & 37 & 7.10 & 9729 & 2192 \\
3.pods & 26 & 5.74 & 3883 & 776 \\
4.icde & 22 & 6.83 & 3307 & 1970 \\
5.er & 16 & 5.80 & 1486 & 1338 \\
6.edbt & 13 & 3.89 & 658 & 434 \\
7.eds & 12 & 3.65 & 527 & 101 \\
8. adbt & 12 & 2.86 & 412 & 42 \\
9.icdt & 11 & 4.79 & 580 & 313 \\
10.oodbs & 11 & 3.96 & 480 & 122 \\
\hline
\end{tabular}

Table 7. Conferences ranking using the contemporary h-index

\begin{tabular}{lccccc}
\hline Name & $h_{c}$ & $a_{c}$ & $h$ & $N_{c, \text { tot }}$ & $N_{p}$ \\
\hline 1. sigmod & 21 & 9.49 & 45 & 12261 & 2059 \\
2.vldb & 17 & 11.34 & 37 & 9729 & 2192 \\
3.pods & 12 & 9.73 & 26 & 3883 & 776 \\
4.icde & 11 & 11.88 & 22 & 3307 & 1970 \\
5.icdt & 8 & 5.04 & 11 & 580 & 313 \\
6.edbt & 7 & 6.16 & 13 & 658 & 434 \\
7. oodbs & 6 & 3.63 & 11 & 480 & 122 \\
8.er & 5 & 16.21 & 16 & 1486 & 1338 \\
9. kdd & 5 & 6.89 & 6 & 243 & 1074 \\
10.dood & 5 & 6.57 & 8 & 440 & 171 \\
\hline
\end{tabular}

\footnotetext{
${ }^{*}$ The symbol $a_{c}$ in Table 6 and the symbol $a_{t}$ in Table 8 correspond to the a-index in Definition 2.
} 
A. SiDIROPOULOS et al.: Generalized Hirsch h-index

Table 8. Conferences ranking using the trend $\mathrm{h}$-index

\begin{tabular}{lccccc}
\hline Name & $h_{t}$ & $a_{t}$ & $h$ & $N_{c, \text { tot }}$ & $N_{p}$ \\
\hline 1. sigmod & 34 & 6.67 & 45 & 12261 & 2059 \\
2.vldb & 27 & 8.00 & 37 & 9729 & 2192 \\
3.pods & 19 & 6.53 & 26 & 3883 & 776 \\
4.icde & 16 & 9.52 & 22 & 3307 & 1970 \\
5.icdt & 12 & 3.67 & 11 & 580 & 313 \\
6. edbt & 9 & 6.02 & 13 & 658 & 434 \\
7.er & 8 & 10.35 & 16 & 1486 & 1338 \\
8. dood & 8 & 4.43 & 8 & 440 & 171 \\
9. kdd & 7 & 6.42 & 6 & 243 & 1074 \\
10.dbpl & 7 & 5.11 & 8 & 410 & 228 \\
\hline
\end{tabular}

Table 9. Conferences ranking using the normalized h-index

\begin{tabular}{lccccc}
\hline Name & $h_{n}$ & $h$ & $a$ & $N_{c, \text { tot }}$ & $N_{p}$ \\
\hline 1.adbt & 0.28 & 12 & 2.86 & 412 & 42 \\
2.dpds & 0.17 & 7 & 2.97 & 146 & 39 \\
3.eds & 0.11 & 12 & 3.65 & 527 & 101 \\
4.icod & 0.11 & 6 & 3 & 108 & 52 \\
5.jcdkb & 0.11 & 8 & 3.32 & 213 & 70 \\
6.ddb & 0.09 & 4 & 6.87 & 110 & 44 \\
7.oodbs & 0.09 & 11 & 3.96 & 480 & 122 \\
8.tdb & 0.08 & 3 & 6.44 & 58 & 36 \\
9.berkeley & 0.07 & 10 & 3.52 & 352 & 142 \\
\hline
\end{tabular}

In Figure 2 we present the progress of selected conferences in the same way we used for scientists. Note here that the h-index is shown per year in the graphs, which means that this is the computed h-index during the specific year. E.g., the h-index that is computed for the VLDB for 1995 is the h-index that is computed, if we exclude everything from our database after 1995. Apparently, this is different from a score for the VLDB'95, which we defined earlier as $h_{1995}{ }^{*}$

Figure 2c presents the history of the SIGMOD conference. According to the tables, SIGMOD is ranked first. In the figure, we observe its steeply ascending line. Also the trend h-index remains higher than the h-index (until 1999). On the other hand, the PODS conference (Figure 2b) follows a bending line after 1993. Thus, the h-index is lightly increased. This can be attributed to the shift in the conference topics; it moved from issues related to the common ground of artificial intelligence and databases, to topics closer to database theory, thus losing some popularity. ICDE is a relatively younger conference compared to the rest of the conferences presented, but we can see in the plot (Figure 2e), that it follows a rapidly ascending course, indicating a very competitive conference.

\footnotetext{
* Due to the lack of citations for the years after 1999, in all graphs there is a stabilization of the h-index line and a downfall for the indicators trend h-index and contemporary h-index.
} 


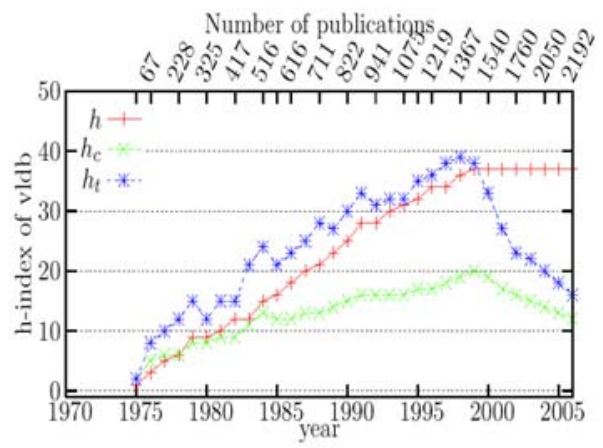

(a)

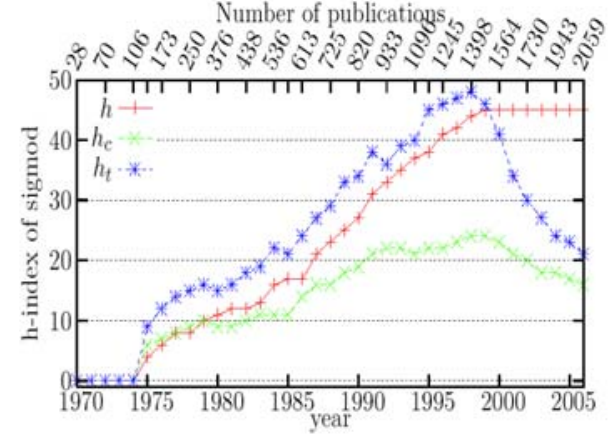

(c)

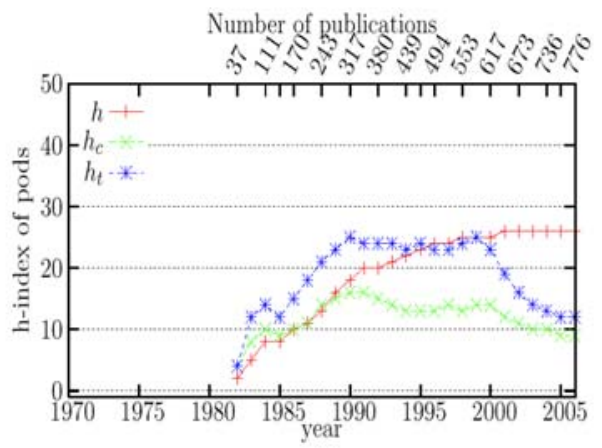

(b)

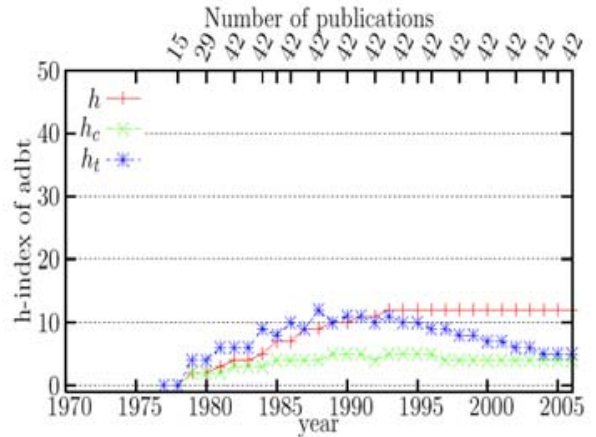

(d)

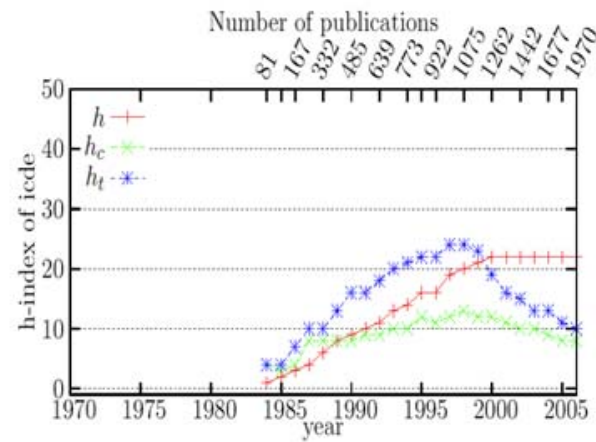

(e)

Figure 2. The h-index of database conferences

Finally, with respect to the ADBT conference (Figure 2d), we mention that this conference was organized only three times (1977, 1979 and 1982). As we can see in the upper $x$ axis, the number of publications stopped increasing after 1982. Thus, we can 
not compare it to the rest of the conferences. The small number of publications of ADBT is the reason that ADBT is ranked first in Table 9.

The next step in conference ranking is to evaluate the usefulness and benefit of Definitions 6 and 7. This way we evaluate, for example, VLDB' 95 independently from VLDB'94. Obviously, in this case it is meaningless to add a second time dimension (with indicators contemporary h-index and trend $\mathrm{h}$-index). The contemporary h-index of VLDB'95 will be stable during all the following years, since all papers are published during the same year. On the other hand, it is not important to see whether a conference organized in 1980 still gets citations.

Indicatively, we present Tables 10 and 11, which contain the conference rankings for the years of 1995 and 1990 respectively. In part (a) of these tables the ordering is performed by using the yearly h-index $\left(h_{y}\right)$. Factor $a$ is the second criterion for the ranking. We also present the columns $h_{y}^{n}$, which is the h-index divided by the number of publications $N_{p, y}$. Also, column $N_{c, 1995}$ is the number of citations to papers published during 1995. In the second part (b) of the tables, the ordering is computed based to the normalized h-index. Notice here, that although it seems to have equivalences by using $h_{y}^{n}$, the real numbers make such a situation almost unprovable (i.e., $5 / 24=0.20833$, $6 / 29=0.206897$ ).

What we observe here is that there are no important differences in the ranking for the two indicative years, even using the normalized h-index. On the other hand, it is obvious that the use of normalized $\mathrm{h}$-index gives a small advantage to the conferences that have a small number of publications. For example, the VLDB conference is almost stable in the first place using the yearly h-index, but it is improbable to get the first place using the normalized yearly h-index.

Table 10. Conferences ranking for the year 1995

\begin{tabular}{lccccc}
\hline \multicolumn{7}{c}{ (a) } \\
\hline \multicolumn{1}{c}{ Name } & $h_{1995}$ & $a$ & $h_{1995}^{n}$ & $N_{c, 1995}$ & $N_{p, 1995}$ \\
\hline 1.vldb & 11 & 3.57 & 0.15 & 432 & 72 \\
2. sigmod & 9 & 4.62 & 0.10 & 375 & 85 \\
3.icde & 6 & 6.63 & 0.08 & 239 & 68 \\
4.pods & 6 & 4.16 & 0.20 & 150 & 29 \\
5.ssd & 5 & 2.08 & 0.20 & 52 & 24 \\
6.kdd & 4 & 3.81 & 0.07 & 61 & 56 \\
7.cikm & 3 & 6.22 & 0.05 & 56 & 55 \\
8.dood & 3 & 5.88 & 0.06 & 53 & 46 \\
9.icdt & 3 & 3.66 & 0.08 & 33 & 34 \\
10.er & 3 & 3.33 & 0.06 & 30 & 47 \\
\hline
\end{tabular}

\begin{tabular}{lccc}
\hline \multicolumn{1}{c}{ Name } & $h_{1995}^{n}$ & $h_{1995}$ & $N_{p, 1995}$ \\
\hline \multicolumn{1}{c}{ 1. ssd } & 0.20 & 5 & 24 \\
2.pods & 0.20 & 6 & 29 \\
3.cdb & 0.2 & 2 & 10 \\
4.vldb & 0.15 & 11 & 72 \\
5.coopis & 0.14 & 3 & 21 \\
6. artdb & 0.11 & 2 & 17 \\
7. sdb & 0.11 & 1 & 9 \\
8. sigmod & 0.10 & 9 & 85 \\
9.ride & 0.10 & 2 & 19 \\
10.tdb & 0.1 & 2 & 20 \\
\hline
\end{tabular}


A. SIDIROPOULOS et al.: Generalized Hirsch h-index

Table 11. Conferences ranking for the year 1990

\begin{tabular}{|c|c|c|c|c|c|}
\hline \multicolumn{6}{|c|}{ (a) } \\
\hline Name & $h_{1990}$ & $a$ & $h_{1990}^{n}$ & $N_{c, 1990}$ & $N_{p, 1990}$ \\
\hline 1. vldb & 16 & 2.57 & 0.26 & 659 & 60 \\
\hline 2. sigmod & 15 & 3.44 & 0.31 & 776 & 48 \\
\hline 3. icde & 11 & 2.76 & 0.16 & 335 & 67 \\
\hline 4. pods & 11 & 2.40 & 0.30 & 291 & 36 \\
\hline 5. edbt & 7 & 2.83 & 0.21 & 139 & 32 \\
\hline 6. icdt & 5 & 4.32 & 0.14 & 108 & 34 \\
\hline 7. dpds & 4 & 3.75 & 0.22 & 60 & 18 \\
\hline 8. er & 3 & 4.66 & 0.08 & 42 & 35 \\
\hline 9. $\mathrm{ds}$ & 3 & 4.11 & 0.12 & 37 & 24 \\
\hline 10. ssdbm & 3 & 3 & 0.16 & 27 & 18 \\
\hline
\end{tabular}

\begin{tabular}{lccc}
\hline \multicolumn{1}{c}{ Name } & $(\mathrm{b})$ & & \\
\hline 1.ssd & $h_{1995}^{n}$ & $h_{1995}$ & $N_{p, 1995}$ \\
2.pods & 0.20 & 5 & 24 \\
3.cdb & 0.20 & 6 & 29 \\
4.vldb & 0.2 & 2 & 10 \\
5.coopis & 0.15 & 11 & 72 \\
6. artdb & 0.14 & 3 & 21 \\
7. sdb & 0.11 & 2 & 17 \\
8. sigmod & 0.11 & 1 & 9 \\
9. ride & 0.10 & 9 & 85 \\
10.tdb & 0.10 & 2 & 19 \\
\hline & 0.1 & 2 & 20 \\
\hline
\end{tabular}

Table 12. Journal ranking using the h-index (left) and normalized h-index (right)

\begin{tabular}{lcccc}
\hline \multicolumn{1}{c}{ Name } & $h$ & $a$ & $N_{c, \text { tot }}$ & $N_{p}$ \\
\hline 1.tods & 49 & 3.88 & 9329 & 598 \\
2.tkde & 18 & 4.69 & 1520 & 1388 \\
3.is & 16 & 4.71 & 1208 & 934 \\
4. sigmod & 15 & 5.07 & 1142 & 1349 \\
5.tois & 13 & 4.37 & 740 & 378 \\
6.debu & 11 & 7.13 & 863 & 877 \\
7.vldb & 9 & 5.03 & 408 & 281 \\
8.ipl & 8 & 6.06 & 388 & 4939 \\
9.dke & 6 & 8.77 & 316 & 773 \\
10.dpd & 6 & 5.25 & 189 & 238 \\
\hline
\end{tabular}

\begin{tabular}{lccccc}
\hline \multicolumn{1}{c}{ Name } & $h_{n}$ & $h$ & $a$ & $N_{c, \text { tot }}$ & $N_{p}$ \\
\hline 1.tods & 0.08 & 49 & 3.88 & 9329 & 598 \\
2.tois & 0.03 & 13 & 4.37 & 740 & 378 \\
3.vldb & 0.03 & 9 & 5.03 & 408 & 281 \\
4.dpd & 0.02 & 6 & 5.25 & 189 & 238 \\
5.jiis & 0.01 & 6 & 4.33 & 156 & 318 \\
6.datamine & 0.01 & 3 & 5.11 & 46 & 162 \\
7.is & 0.01 & 16 & 4.71 & 1208 & 934 \\
8.ijcis & 0.01 & 4 & 3.12 & 50 & 255 \\
9.tkde & 0.01 & 18 & 4.69 & 1520 & 1388 \\
10.debu & 0.01 & 11 & 7.13 & 863 & 877 \\
\hline
\end{tabular}

Table 13. Journal ranking using the contemporary h-index (left) and trend h-index (right)

\begin{tabular}{lccccc}
\cline { 1 - 4 } \multicolumn{1}{c}{ Name } & $h_{c}$ & $a_{c}$ & $h$ & $N_{c, \text { tot }}$ & $N_{p}$ \\
\hline 1.tods & 18 & 6.25 & 49 & 9329 & 598 \\
2.tkde & 10 & 6.40 & 18 & 1520 & 1388 \\
3. sigmod & 9 & 6.17 & 15 & 1142 & 1349 \\
4.debu & 6 & 9.21 & 11 & 863 & 877 \\
5.vldb & 6 & 6.47 & 9 & 408 & 281 \\
6.tois & 6 & 6.09 & 13 & 740 & 378 \\
7.is & 5 & 12.77 & 16 & 1208 & 934 \\
8.dpd & 5 & 4.19 & 6 & 189 & 238 \\
9.jiis & 5 & 3.79 & 6 & 156 & 318 \\
10.dke & 4 & 7.70 & 6 & 316 & 773 \\
\hline
\end{tabular}

\begin{tabular}{lccccc}
\hline \multicolumn{1}{c}{ Name } & $h_{t}$ & $a_{t}$ & $h$ & $N_{c, \text { tot }}$ & $N_{p}$ \\
\hline 1.tods & 28 & 4.93 & 49 & 9329 & 598 \\
2.tkde & 13 & 6.64 & 18 & 1520 & 1388 \\
3. sigmod & 12 & 5.85 & 15 & 1142 & 1349 \\
4.vldb & 10 & 3.75 & 9 & 408 & 281 \\
5.is & 9 & 7.11 & 16 & 1208 & 934 \\
6.debu & 9 & 6.98 & 11 & 863 & 877 \\
7.tois & 9 & 4.83 & 13 & 740 & 378 \\
8.dpd & 6 & 4.88 & 6 & 189 & 238 \\
9.jiis & 6 & 4.75 & 6 & 156 & 318 \\
10.dke & 5 & 8.18 & 6 & 316 & 773 \\
\hline
\end{tabular}

In Figure 3 we present the plots for the values of yearly h-index $\left(h_{y}\right)$ and normalized yearly h-index $\left(h_{y}^{n}\right)$ for the top four conferences VLDB, PODS, SIGMOD and ICDE. The values for $h_{y}$ are drawn using bars, because each value is independent from the rest 
of them. The value for $h_{y}$ of a conference has different upper bound for each year. The upper bound for each year is defined by the number of papers published during this year. This is depicted on the upper $x$ axes. On the other hand, the $h_{y}^{n}$ values are normalized. So, it is a comparable value for the two years of a conference and it is drawn with the cross points line. The values for the $h_{y}^{n}$ index are presented in axes $y 2$. There is no association of axes $y 1$ to $y 2$, thus we cannot compare (obviously) the values of $h_{y}^{n}$ to $h_{y}$. The only remark that we can make is that the one curve follows approximately the other. This comes in agreement with the conclusions derived from Tables 10 and 11 .

Experiments with journals ranking. In the case of journals, we can use the basic form of h-index, as well as the generalizations contemporary $\mathrm{h}$-index and trend $\mathrm{h}$-index and the variant normalized h-index we defined for scientists and for conferences. Here, similarly to the case of conferences, the normalized h-index is a valuable indicator contrary to the case of the scientists.

Tabular data in Tables 12, 13 present the top-10 journals according to the four aforementioned indices. As expected, the ACM TODS (tods), IEEE TDKE (tkde), SIGMOD Record (sigmod) are the top three journals. The striking observation is that the Information Systems (is) drops in the ranking with the contemporary h-index and trend h-index as compared to its position with h-index, implying that it is not considered an exceptionally prestigious journal anymore. On the contrary, SIGMOD Record and VLDB Journal (vldb) show an uprising trend.

In Figure 4 we present the results of computing the defined indices for the major journals of the database domain on a per year basis. Due to the lack of available data after the year 2000, all indices drop steeply. Though, the case of ACM TODS is worthwhile mentioning. Its trend h-index drops after 1993, which can be attributed to the relatively large end-to-end publication time of its articles during the years 1990-2000,39 which acted as an impediment for the authors to submit their works in that venue. Fortunately, this is not the case anymore. Also, the case of SIGMOD Record is characteristic, because, even though it has been published since 1970, its indices get really noticeable only after 1980 , when this newsletter started to publish some very good survey-type articles and was freely available on the Web, which improved its visibility. 


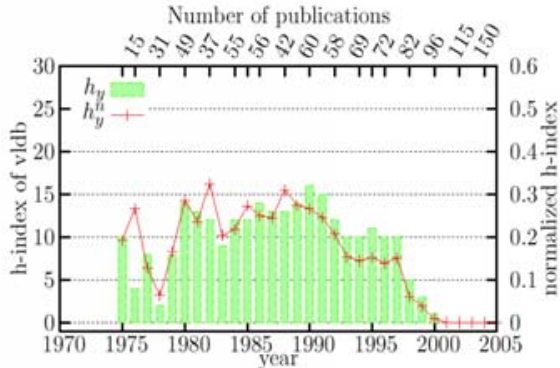

(a)

Number of publications

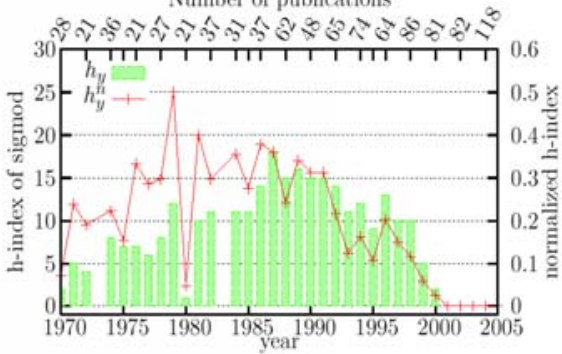

(c)

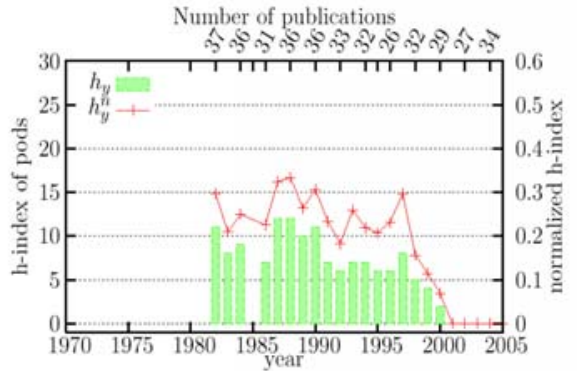

(b)

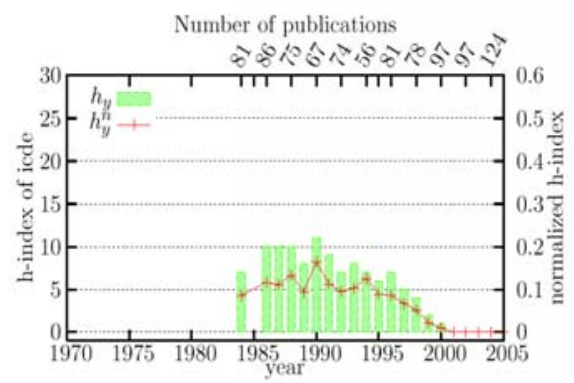

(d)

Figure 3. The yearly h-index and normalized yearly h-index of database conferences

Finally, in Figure 5 we present the results of computing the yearly h-index and the normalized yearly h-index for the major journals of the database domain on a per year basis. In these figures we can easily see that the ACM TODS journal undoubtedly gets the first place. Also, Figure 5a shows that the yearly h-index follows a decreasing path which comes in agreement with Figure 4a. Also, Figure 5e comes in agreement with Figure 4e. In Figure 5c it is shown that the small number of the published papers for the years 1984 and 1986 make the normalized yearly h-index to get higher than usually for the SIGMOD Record journal. TKDE (Figure 5b) seems to follow a descreasing slope and finally, VLDB (Figure 5d) follows an uprising trend until 1996. 


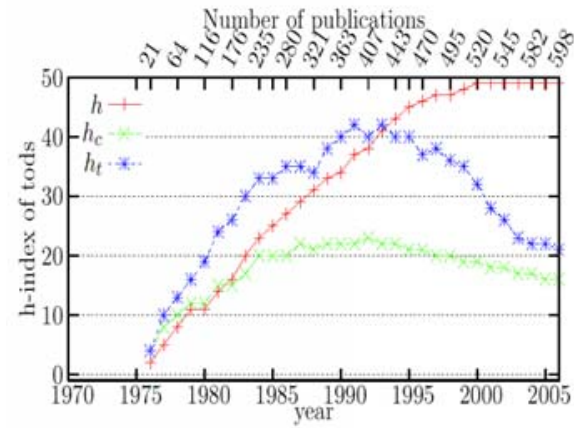

(a)

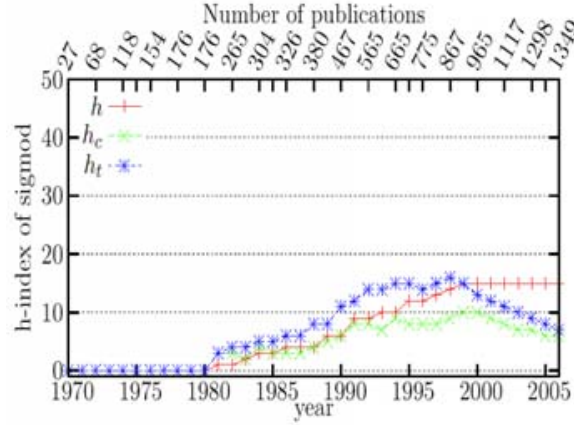

(c)

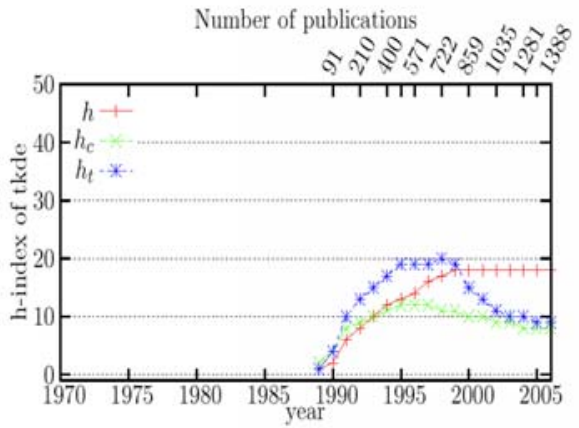

(b)

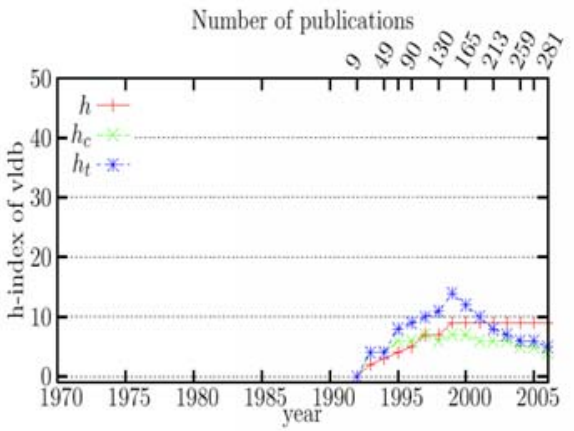

(d)

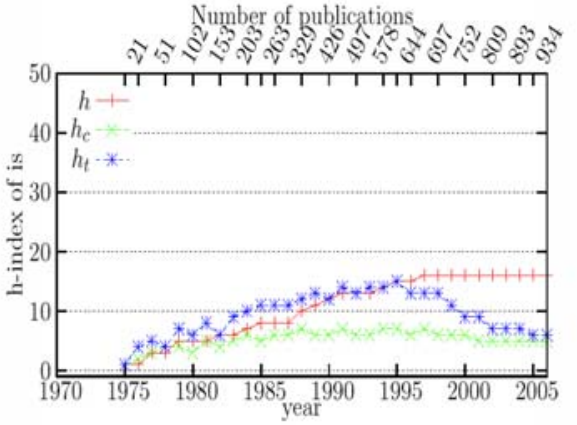

(e)

Figure 4. The h-index, contemporary h-index and trend h-index of major database journals 


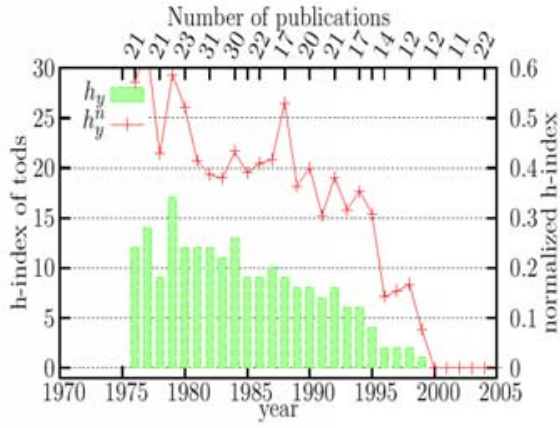

(a)

Number of publications

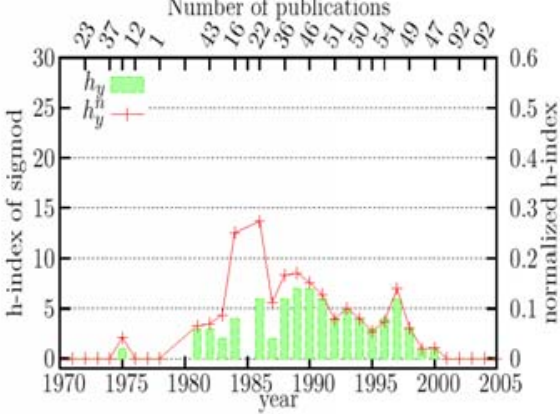

(c)

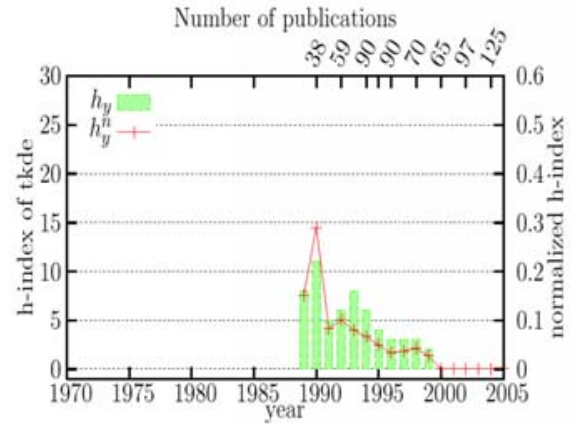

(b)

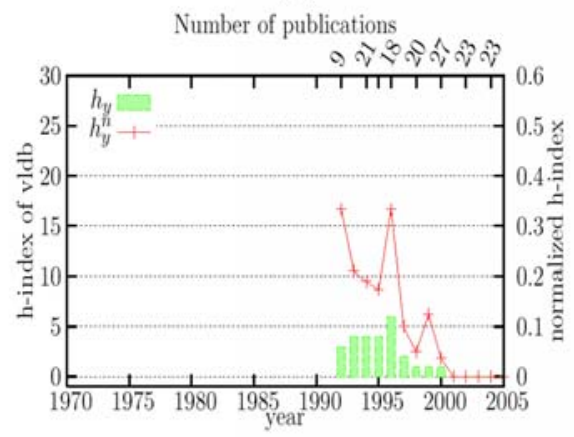

(d)

Number of publications

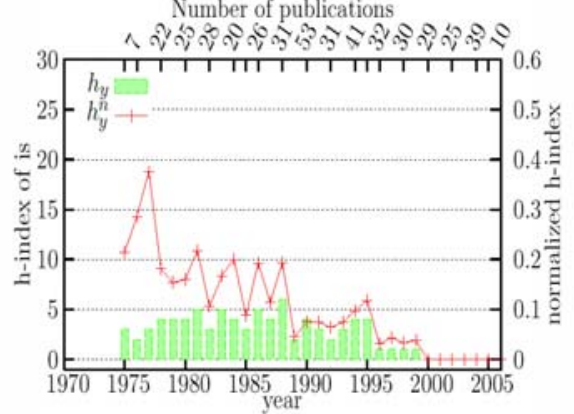

(e)

Figure 5. The yearly h-index and the normalized yearly h-index of major database journals 


\section{Conclusions}

Estimating the significance of a scientist's work is a very important issue for prize awarding, faculty recruiting, etc. This issue has received a lot of attention during the last years and a number of metrics have been proposed which are based on arithmetics upon the number of articles published by a scientist and the total number of citations to these articles. The interest on these topics has been renewed and in a path-breaking paper, Hirsch proposed the h-index to perform fair ranking of scientists, avoiding many of the drawbacks of the earlier bibliographic ranking methods.

The initial proposal and meaning of the h-index has various shortcomings, mainly of its inability to differentiate between active and inactive (or retired) scientists and its weakness to differentiate between significant works in the past (but not any more) and the works which are "trendy" or the works which continue to shape the scientific thinking.

Based on the identification of these shortcomings of h-index, we proposed in this article a number of effective h-index generalizations and some variants. Some of these novel citation indices aim at the ranking of scientists by taking into account the age of the published articles, or the age of the citations to each article. The other citation indices aim at ranking publication venues, i.e., conferences and journals, taking into account the variable number of the published articles.

To evaluate the proposed ranking metrics, we conducted extensive experiments on an online bibliographic database containing data from journal and conference publications as well, and moreover focused in the specific area of databases. From the results we obtained, we concluded that h-index is not a general purpose indicative metric. Some of the novel indices, namely contemporary h-index and trend h-index, are able to disclose latent facts in citation networks, like trendsetters and brilliant young scientists. For the case of conference and journal ranking, the indices normalized hindex, contemporary h-index and trend h-index give a more fair view for the ranking. Finally, the yearly h-index and the normalized yearly h-index can be used in order to evaluate separately each conference/journal success.

This paper is a significantly expanded version of a short paper which appeared in the Proceedings of the 4th ACM International Workshop on Link Analysis: Dynamics and Static of Large Networks (LinkKDD) (In conjunction with ACM KDD), ACM Press, Philadelphia, PA, USA, August 20, 2006. 


\section{References}

1. P. BALL, Index aims for fair ranking of scientists - h-index sums up publication record. Nature, 436 (7053) (2005) 900 .

2. M. G. BANKS, An extension of the Hirsch index: Indexing scientific topics and compounds. Scientometrics, 69 (1) (2006) 161-168.

3. J. BAR-ILAN, h -index for price medalists revisited. ISSI Newsletter 5 (Jan. 2006).

4. S. J. BARNES, Assessing the value of IS journals. Communications of the ACM, 48 (1) (2005) 110-112.

5. P. D. Batista, M. G. Campiteli, O. Kinouchi, A. S. Martinez, Is it possible to compare researchers with different scientific interests? Scientometrics, 68 (1) (2006) 179-189.

6. P. A. Bernstein, E. Bertino, A. Heuer, C. J. Jensen, H. Meyer, M. Tamer Ozsu, R. T. SNODGRASS, K.-Y. WHANG, An apples-to-apples comparison of two database journals. ACM SIGMOD Record, 34 (4) (2005) 61-64.

7. P. BhARATI, P. TARASEWICH, Global perceptions of journals publishing e-commerce research. Communications of the ACM, 45 (5) (2002) 21-26.

8. J. Bollen, M. A. Rodriguez, H. VAN DE SOMPEL, Journal status, (May 2006), Preprint available at http://arxiv.org/abs/cs/0601030

9. L. BORNMANN, H.-D. DANIEL, Does the h-index for ranking of scientists really work? Scientometrics, 65 (3) (2005) 391-392.

10. T. Braun,W. GlänZel, A. Schubert, A Hirsch-type index for journals. Scientometrics, 69 (1) (2006) 169-173.

11. B. Cronin, L. Meho, Using the H-index to rank influential information scientists. Journal of the American Society for Information Science and Technology, 57 (9) (2006) 1275-1278.

12. L. EGgHe, Dynamic h-index: The Hirsch index in function of time. Scientometrics, (2006), To appear.

13. L. EGGHE, Theory and practise of the g-index. Scientometrics, 69 (1) (2006) 131-152.

14. L. EgGhe, R. Rousseau, An informetric model for the Hirsch-index. Scientometrics, 69 (1) (2006) 121-129.

15. E. Elmacioglu, D. LeE, On six degrees of separation in DBLP-DB and more. ACM SIGMOD Record, 34 (2) (2005) 33-40.

16. E. GARFIELD, Citation analysis as a tool in journal evaluation. Science, 178 (1972) 471-479.

17. W. GLÄNZEL, On the h-index - A mathematical approach to a new measure of publication activity and citation impact. Scientometrics, 67 (2) (2006) 315-321.

18. B. C. Hardgrave, K. A. Walstrom, Forums for MIS scholars. Communications of the ACM, 40 (11) (1997) 119-124.

19. J. E. HiRSCH, An index to quantify an individual's scientific research output. Proceedings of the National Academy of Sciences, 102 (46) (2005) 16569-16572.

20. C. W. Holsapple, H. Johnson, L. E. Manakyan, J. TANner, Business computing research journals: A normalized citation analysis. Journal of Management Information Systems, 11 (1) (1994) 131-140.

21. P. Katerattanakul, B. T. Han, S. Hong, Objective quality ranking of computing journals. Communications of the ACM, 46 (10) (2003) 111-114.

22. R. Kelly Rainer, M. D. Miller, Examining differences across journal rankings. Communications of the ACM, 48 (2) (2005) 91-94.

23. J. KleINBERG, Authoritative sources in a hyperlinked environment. Journal of the ACM, 46 (5) (1999) 604-632.

24. L. LIANG, h-index sequence and h-index matrix: Constructions and applications. Scientometrics, 69 (1) (2006) 153-159.

25. P. LOWRY, D. Romans, A. CuRTIS, Global journal prestige and supporting disciplines: A scientometric study of information systems journals. Journal of the Association for Information Systems, 5 (2) (2004) 29-75.

26. N. A. Mylonopoulos, V. TheOharakis, Global perception of IS journals. Communications of the $A C M, 44$ (9) (2001) 29-33. 
A. SIDIROPOULOS et al.: Generalized Hirsch h-index

27. M. A. NASCimento, J. SANDER, J. Pound, Analysis of SIGMOD's co-authorship graph. ACM SIGMOD Record, 32 (3) (2003) 8-10.

28. S. P. Nerur, R. Sikora, G. Mangalaraj, V. Balijepally, Assessing the relative influence of journals in a citation network. Communications of the ACM, 48 (11) (2005) 71-74.

29. L. Page, S. Brin, R. Motwani, T. Winograd, The PageRank citation ranking: Bringing order to the Web. Technical Report TR-1999-66, Stanford University,1999.

30. A. F. J. VAN RAAN, Comparison of the Hirsch-index with standard bibliometric indicators and with peer judgment for 147 chemistry research groups. Scientometrics, 67 (3) (2006) 491-502.

31. E. RAHM, A. Thor, Citation analysis of database publications. ACM SIGMOD Record, 34 (4) (2005) 48-53.

32. R. RousseAu, A case study: Evolution of JASIS 'Hirsch index. Library and Information Science, Jan. 2006. Available at http://eprints.rcils.org/archive/00005430

33. R. RousSEAU, Simple models and the corresponding $\mathrm{h}$ and $\mathrm{g}$ index. Library and Information Science, Apr. 2006. Available at http://eprints.rcils.org/archive/00006153

34. G. SAAD, Exploring the h-index at the author and journal levels using bibliometric data of productive consumer scholars and business-related journals respectively. Scientometrics, 69 (1) (2006) 117-120.

35. R. B. Schwartz, M. C. Russo, How to quickly find articles in the top IS journals. Communications of the ACM, 47 (2) (2004) 98-101.

36. A. Sidiropoulos, Y. MAnolopoulos, A citation-based system to assist prize awarding. $A C M$ SIGMOD Record, 34 (4) (2005) 54-60.

37. A. Sidiropoulos, Y. MANOlopoulos, A new perspective to automatically rank scientific conferences using digital libraries. Information Processing \& Management, 41 (2) (2005) 289-312.

38. A. Sidiropoulos, Y. MANOlOpOUlos, Generalized comparison of graph-based ranking algorithms for publications and authors. Journal for Systems and Software (2006), To appear.

39. R. SNODGRASS, Journal relevance. ACM SIGMOD Record, 32 (3) (2003) 11-15. 